\title{
ASSOCIATION OF REDUCED EXTRACELLULAR BRAIN AMMONIA, LACTATE, AND INTRACRANIAL PRESSURE IN PIGS WITH ACUTE LIVER FAILURE

\author{
Christopher Rose ${ }^{1,2}$, Lars M. Ytrebø ${ }^{3}$, Nathan A. Davies ${ }^{4}$, Sambit Sen $^{4}$, Geir I. Nedredal ${ }^{3}$, \\ Mireille Belanger ${ }^{2}$, Arthur Revhaug ${ }^{3}$ and Rajiv Jalan ${ }^{4, \S *}$
}

1. Department of Cellular Neuroscience, Max-Delbrück Center for Molecular Medicine, Berlin, Germany

2. Neuroscience Research Unit, Hôpital Saint-Luc, Montreal, Quebec, Canada

3. Department of Digestive Surgery, University Hospital of North Norway, University of Tromso, Norway

4. Liver Failure Group, Institute of Hepatology, Division of Medicine, University College London, London, UK

\begin{abstract}
${ }^{\dagger}$ This work is part of an international multicenter collaboration studying various end-organ functions in acute liver failure. Different pathophysiological aspects will be dealt with in separate articles. This article does not contain data published or submitted elsewhere. ${ }^{*}$ Potential conflict of interest: Nothing to report.
\end{abstract}

\section{ABSTRACT}

We previously demonstrated in pigs with acute liver failure (ALF) that albumin dialysis using the molecular adsorbents recirculating system (MARS) attenuated a rise in intracranial pressure (ICP). This was independent of changes in arterial ammonia, cerebral blood flow and inflammation, allowing alternative hypotheses to be tested. The aims of the present study were to determine whether changes in cerebral extracellular ammonia, lactate, glutamine, glutamate, and energy metabolites were associated with the beneficial effects of MARS on ICP. Three randomized groups [sham, ALF (induced by portacaval anastomosis and hepatic artery ligation), and ALF+MARS] were studied over a 6-hour period with a 4-hour MARS treatment given beginning 2 hours after devascularization. Using cerebral microdialysis, the ALF-induced increase in extracellular brain ammonia, lactate, and glutamate was significantly attenuated in the ALF+MARS group as well as the increases in extracellular lactate/pyruvate and lactate/glucose ratios. The percent change in extracellular brain ammonia correlated with the percent change in ICP $\left(\boldsymbol{r}^{2}=0.511\right)$. Increases in brain lactate dehydrogenase activity and mitochondrial complex activity for complex IV were found in ALF compared with those in the sham, which was unaffected by MARS treatment. Brain oxygen consumption did not differ among the study groups. Conclusion: The observation that brain oxygen consumption and mitochondrial complex enzyme activity changed in parallel in both ALFand MARS-treated animals indicates that the attenuation of increased extracellular brain ammonia (and extracellular brain glutamate) in the MARS-treated animals reduces energy demand and increases supply, resulting in attenuation of increased extracellular brain lactate. The mechanism of how MARS reduces extracellular brain ammonia requires further investigation. (HEPATOLOGY 2007.)

\section{Abbreviations}

MARS, molecular adsorbents recirculating system; ALF, acute liver failure; LDH, lactate dehydrogenase; ICP, intracranial pressure.

\section{INTRODUCTION}

Acute liver failure (ALF) is defined by the occurrence of hepatic encephalopathy (HE), which is characterized by an increase in intracranial pressure (ICP) that may lead to cerebral herniation, an important cause of death. ${ }^{1}$ Current hypotheses suggest that increased ICP in ALF is the result of multiple factors that involve the effects of hyperammonemia, increased cerebral blood flow, and inflammation. ${ }^{2}$ Increased brain ammonia, arising from the onset of 
hyperammonemia, is detrimental to neurological function. ${ }^{3}$ Arterial ammonia levels, ammonia brain delivery, and metabolic rate correlate with severity of intracranial hypertension and risk of cerebral herniation. ${ }^{1,4,5}$

Alterations in brain energy metabolism have been hypothesized to be involved in the cerebral consequences of ALF because ammonia is known to inhibit the rate-limiting enzyme $\alpha$-ketoglutarate dehydrogenase in the tricarboxylic acid cycle (TCA). ${ }^{6}$ This is supported by experiments demonstrating an increase in de novo synthesis of lactate ${ }^{7,8}$ from glucose (using nuclear magnetic resonance spectroscopy) in the brains of rats with ALF. As a result, this would lead to less adenosine triphosphate (ATP) production per molecule of glucose compared with that in aerobic metabolic pathways (through TCA cycle). Therefore, increased ammonia in the brain reduces generation of ATP. On the other hand, elevated ammonia also increases energy demand. Ammonia detoxification through glutamine synthetase activity and stimulation of glutamate uptake by ammonia into astrocytes ${ }^{9}$ along with activation of NMDA receptors ${ }^{10}$ (both resulting in increased $\mathrm{Na}^{+}-\mathrm{K}^{+}$-adenosine triphosphatase [ATPase] activity) all require ATP. Thus, ammonia affects brain bioenergetics, through both an increase in the demand for and a reduction in the supply of ATP.

Albumin dialysis, using the molecular adsorbents recirculating system (MARS), removes protein bound substances from the blood. Several controlled clinical trials have shown that MARS therapy is an effective treatment for HE. ${ }^{11-16}$ The mechanism by which MARS treatment results in improved HE is not clear, as results showing a reduction in blood ammonia with MARS have been inconsistent. In keeping with the results in humans, we recently showed attenuation in the rise in ICP (following a 4-hour MARS treatment) in a porcine model of ALF (induced by liver devascularization). ${ }^{17}$ This beneficial effect of MARS was not associated with any significant changes in arterial ammonia, cerebral blood flow, or severity of inflammation, ${ }^{17}$ allowing the use of this model and MARS therapy as an experimental tool to explore alternative pathophysiological mechanisms that may be important in the pathogenesis of intracranial hypertension in ALF. The aims of the present study were to determine whether changes in ammonia, glutamine, glutamate, and energy metabolites in the extracellular compartment of the brain are associated with the changes in ICP previously observed in ALF- and ALF+MARS-treated pigs. ${ }^{17}$

\section{MATERIALS AND METHODS}

The study was performed in the Surgical Research Laboratory at the University of Troms $\emptyset$, Norway, and was approved by the Norwegian Experimental Animal Board. Twenty-four female Norwegian Landrace pigs from 3 litters weighing $23-30 \mathrm{~kg}$ (mean weight \pm SEM: $26.8 \pm 0.3 \mathrm{~g}$ ) were used.

\section{Study Design.}

The pigs were randomized into 3 groups 17: sham, ALF, and ALF+MARS (8 per group). ALF was induced by devascularization of the liver. This was achieved with an end-to-side portacaval anastomosis followed by hepatic artery ligation (HAL). In the sham group, a laparotomy was performed without interfering with hepatic blood supply. Details of the operation were described previously. ${ }^{18,19}$ Time $(\boldsymbol{T})=0$ was defined as following HAL in the ALF and ALF+MARS groups or just prior to closing the abdominal wall in the sham group. All pigs were monitored over 6 hours. In the ALF+MARS group, a 4-hour MARS dialysis treatment began at $\boldsymbol{T}=2$ hours. Experiments were terminated at $\boldsymbol{T}=6$ hours by giving the pigs an overdose of pentobarbital and potassium chloride.

\section{Animal Model.}

The animal room facilities, anesthesia, and surgical preparation have previously been described in detail. ${ }^{12,17-20}$ The pigs were premedicated with an intramuscular injection of ketamine $(20 \mathrm{mg} / \mathrm{kg})$ and atropine $(1 \mathrm{mg})$. To prevent any preoperative dehydration, all animals received $500 \mathrm{~mL}$ of $0.9 \% \mathrm{NaCl}$ containing $625 \mathrm{mg}$ of glucose. The pigs were anesthesized with an intravenous bolus of $10 \mathrm{mg} / \mathrm{kg}$ pentobarbital and $10 \mathrm{mg} / \mathrm{kg}$ fentanyl and were maintained during the operation with a central venous infusion of $4 \mathrm{mg}$ pentobarbital $/ \mathrm{kg}$ per hour, $0.02 \mathrm{mg}$ fentanyl $/ \mathrm{kg}$ per hour, and $0.3 \mathrm{mg}$ midazolam $/ \mathrm{kg}$ per hour. Anesthesia was terminated following HAL, and small bolus doses of fentanyl and midazolam 
were given when clinical signs of light sedation appeared. Becuase of drug removal by MARS, 12 animals were kept sedated with a continuous infusion of $0.04 \mathrm{mg}$ fentanyl $/ \mathrm{kg}$ per hour and $0.6 \mathrm{mg}$ midazolam $/ \mathrm{kg}$ per hour.

The pigs were ventilated $\left(\mathrm{PaCO}_{2} 4.5-5.0 \mathrm{kPa}\right)$ throughout the experiment and infused with $0.9 \% \mathrm{NaCl}, 5 \%$ glucose, and $20 \%$ human albumin (to counteract intra-abdominal proteinaceous fluid loss during and after surgery) as described previously. $12,17-20$ Normal core body temperature was maintained at $38.5^{\circ} \mathrm{C} \pm 1{ }^{\circ} \mathrm{C}$. Heparin was used to keep the activated clotting time $($ ACT $)>100$ seconds ( $>180$ seconds during MARS).

\section{Catheter Placement.}

A very thin catheter ${ }^{21}$ was introduced into the abdominal aortas of the pigs in all 3 groups. MARS was performed through an 11.5Fr dual-lumen catheter (Mahurkar, Tyco Healthcare, UK) in the inferior vena cava. To be comparable, ALF and sham animals also received a vena caval catheter.

\section{MARS.}

MARS (Monitor 1, Teraklin AG, Rostock, Germany) ${ }^{2,22,23}$ works on the basis that blood is dialyzed across an albuminimpregnated membrane with $20 \%$ human albumin in the dialysate. Albumin-bound toxins detach from the blood, bind to free-binding sites on the membrane, and then bind to the albumin in the dialysate. The dialysate is then activated through the charcoal and anion-exchanger (adsorbing albumin-bound toxins). The "regenerated" albumin is then recirculated, allowing for removal of more toxins from the blood. ${ }^{22,23}$ A roller pump (Stöckert Shiley, Munich, Germany) and the MARS pump perfusing the blood and albumin dialysate were running at the same rate $(150 \mathrm{~mL} / \mathrm{minute})$.

\section{Microdialysis.}

A burr hole was created over the right frontal region of the skull $(1 \mathrm{~cm}$ lateral, $2 \mathrm{~cm}$ rostral from bregma, and $0.5 \mathrm{~cm}$ ventral), the dura mater was incised, and a microdialysis catheter [CMA 70: 10-mm-long semipermeable membrane (20,000 Da cutoff) and an outer diameter of $0.6 \mathrm{~mm}$ ] was placed into the cortex at least 1 hour before $\boldsymbol{T}=0$. The burr hole was sealed with bone wax to ensure stable positioning of the catheter and to prevent pressure release. The microdialysis catheter was connected to a microinjection pump (CMA/106 microinjection pump; CMA Microdialysis $\mathrm{AB}$, Stockholm, Sweden) and perfused with artificial CSF $\left(\mathrm{Na}^{+} 147 \mathrm{mmol} / \mathrm{L} ; \mathrm{K}^{+} 4 \mathrm{mmol} / \mathrm{L} ; \mathrm{Ca}^{2+} 2.3 \mathrm{mmol} / \mathrm{L} ; \mathrm{Cl}^{-} 156\right.$ $\mathrm{mmol} / \mathrm{L}$ ) at a flow rate of $2.0 \mu \mathrm{L} /$ minute. The microdialysate was collected in microvials every hour, resulting in $120 \mu \mathrm{L}$ for biochemical analysis. Samples were stored at $-20^{\circ} \mathrm{C}$. At the end of each experiment, a craniotomy was performed, and the brain was removed, dissected and examined for any intracranial hemorrhaging.

\section{Extracellular Brain Glucose, Glutamate, Lactate, and Pyruvate Measurements.}

The microdialysis samples were analyzed using a CMA 600 Microdialysis Analyzer (CMA Microdialysis AB). The analyzer measures glucose, lactate, pyruvate, and glutamate concentrations using small-volume samples. The methodologies are based on enzymatic oxidation and colorimetric measurements (CMA Microdialysis AB).

\section{Extracellular Brain Glutamine Measurement.}

Glutamine from microdialysis samples were measured and analyzed using a Perkin-Elmer reverse-phase HPLC system with fluorescence detection and precolumn $\boldsymbol{o}$-phthalaldehyde derivatization. ${ }^{24}$

\section{Extracellular Brain Ammonia Measurement.}

Microdialysate ammonia level was determined using an ammonia reagent kit (Sigma-Aldrich, St. Louis, MO), which was used in conjunction with an automated enzymatic method (Cobas Fara II, Roche, Basel, Switzerland).

\section{Microdialysis Recovery Rates.}

Using the same microdialysis probes and microperfusion pump (at a flow rate of $2.0 \mu \mathrm{L} /$ minute), we measured in vitro the recovery rate for each microdialysate analyte measured. This was done by immersing the probe in a solution of known concentrations of glucose, glutamate, glutamine, lactate, and pyruvate and measuring the concentrations of all 5 analytes in the collected microdialysate. The recovery rate at $2.0 \mu \mathrm{L} /$ minute was $46.7 \% \pm 7.1 \%$ for all analytes. 


\section{Arterial Lactate Measurement.}

Arterial blood was collected every 2 hours and centrifuged, and the plasma was stored at $-70^{\circ} \mathrm{C}$ for subsequent analysis. Arterial plasma lactate level was determined using a lactate reagent kit (Sigma-Aldrich, St. Louis, MO) with an automated enzymatic method (Cobas Fara II, Roche, Basel, Switzerland).

\section{Lactate Dehydrogenase Activity.}

Following termination of experiments, the brain was rapidly removed, and samples were dissected from the frontal cortex and homogenized at $4^{\circ} \mathrm{C}$ in phosphate-buffered saline containing a protease inhibitor cocktail (Sigma-Aldrich, St. Louis, $\mathrm{MO}$ ). Homogenates were vortexed, set on ice for 10 minutes, and then centrifuged at $10,000 \mathrm{~g}$ for 5 minutes at $4^{\circ} \mathrm{C}$.

Lactate dehydrogenase (LDH) activity was assayed in supernatant within 24 hours, and samples were kept at $4^{\circ} \mathrm{C}$ at all times. Cell suspensions were mixed with NADH (0.13 mM; Sigma-Aldrich, St. Louis, MO) and concentrations of pyruvic acid (Sigma-Aldrich, St. Louis, MO) ranging from 0.01 to $1 \mathrm{mM}$ in a $0.1 \mathrm{M}$ potassium phosphate buffer $(\mathrm{pH}=$ 7.4). $\mathrm{LDH}$ activity was assessed over a 2 -minute period by following the rate of oxidation of NADH as measured by spectrophotometric absorbance at $340 \mathrm{~nm}$.

\section{Oxygen Consumption.}

Oxygen consumption was calculated from the blood gas measurements (Rapidab 800, Chiron Diagnostics, MD) in samples collected from catheters inserted into the carotid artery and a separate catheter inserted in the reverse direction with its tip in the jugular bulb. The sampling from this catheter represents the venous drainage from the brain. Extraction was calculated as a percentage using the formula $\left[\left(\mathrm{CaO}_{2}-\mathrm{CvO}_{2}\right) / \mathrm{CaO}_{2}\right] \times 100$.

\section{Mitochondrial Complex Activities.}

Mitochondrial complex activity was measured by well-described spectrophotometric methods (Agilent 8543 Diode array spectrophotometer, Agilent Technologies, UK). ${ }^{25}$ Tissue samples were homogenized on ice with a handheld glass homogenizer, then underwent 3 episodes of rapid freeze-thawing to ensure cell lysis. Complex activity was measured as the inhibitor sensitive rates (rotenone for complex I, antimycin A for complexes II and III, and cyanide for complex IV). To correct for mitochondrial enrichment in the sample, results are expressed as a ratio of citrate synthase activity. ${ }^{26}$

\section{Cytochrome c Oxidase.}

Cytochrome $\boldsymbol{c}$ oxidase concentration in the tissue homogenate was determined spectrophotometrically as the reduced cyanide complex. Briefly, a sample of homogenate was incubated in a phosphate buffer solution (100 mM, pH 7.1) containing sodium ascorbate $(30 \mathrm{mM})$, TMPD $(0.15 \mathrm{mg} / \mathrm{mL})$, and potassium cyanide $(30 \mathrm{mM})$. After 2 minutes, absorbance was measured at $605 \mathrm{~nm}$, and the concentration was calculated using an extinction coefficient of 24 $\mathrm{mM}^{-1} \mathrm{~cm}^{-1}$.

\section{Statistics.}

Results are expressed as mean \pm SEM. Significance of differences within a group was tested by the paired Student $t$ test, and the significance of differences between groups was measured by 1- or 2-way analysis of variance (ANOVA) where applicable. A difference with a $\boldsymbol{P}$ value $<0.05$ was considered statistically significant. GraphPad Prism 4.0 (GraphPad Software, San Diego, CA) was used for statistical analyses.

\section{RESULTS}

As previously mentioned, 173 pigs were excluded (1 sham and 1 ALF because of intracranial hemorrhage and 1 ALF because of technical problems with ICP monitoring), leaving a total of 21 pigs analyzed: 7 sham, 6 ALF, and 8 ALF+MARS.

\section{Extracellular Brain Ammonia and Lactate.}


Two hours following the onset of liver devascularization (pre-MARS treatment), a significant increase in extracellular brain ammonia was observed in pigs with ALF compared with sham-operated control pigs (Fig. 1A). Extracellular ammonia continued to rise in pigs with ALF, whereas this rise was attenuated at $\boldsymbol{T}=6$ hours following a 4-hour treatment with MARS $(\boldsymbol{P}<0.01$; Fig. 1A). Transforming the data into percent increase (from $\boldsymbol{T}=0$ in each group), an earlier attenuation with MARS was observed, as early as 2 hours following MARS treatment (at $\boldsymbol{T}=4-6$; Fig. 1B). To emphasize the effect of MARS on extracellular ammonia, the percent change in extracellular brain ammonia was calculated from $\boldsymbol{T}=2$ hours (start of the MARS treatment) until $\boldsymbol{T}=6$ hours (end of the MARS treatment), as shown in Fig. 1C. Using all 6 times from all 3 groups, a significant correlation was calculated between percent change in extracellular ammonia and percent change in $\operatorname{ICP}\left(\boldsymbol{r}^{2}=0.511, \boldsymbol{P}<0.001\right.$; Fig. 1D).
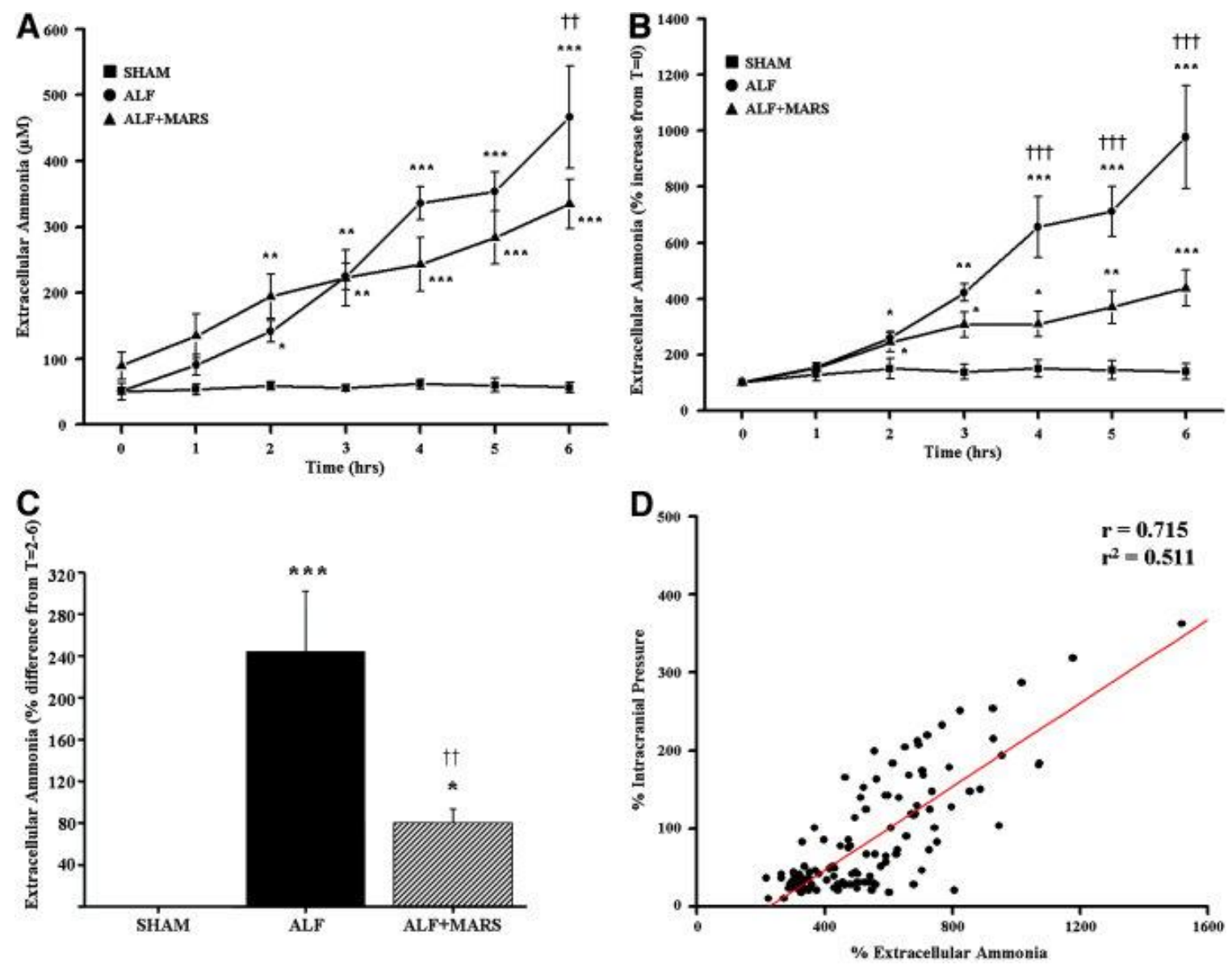

Figure 1. (A) Extracellular brain concentrations of ammonia following induction of ALF $(\boldsymbol{T}=0)$. (B) Percent increase [from baseline $(\boldsymbol{T}=0)$ ] of extracellular concentrations of ammonia. (C) Percent change (from $\boldsymbol{T}=2-6$ ) in extracellular ammonia. (D) Correlation between change in extracellular brain ammonia and change in ICP $\left(* * * \boldsymbol{P}<0.001, * * \boldsymbol{P}<0.01, * \boldsymbol{P}<0.05\right.$ versus SHAM; ${ }^{\dagger \dagger} \boldsymbol{P}<0.001,{ }^{\dagger \dagger} \boldsymbol{P}<0.01$, ALF versus MARS; 1-way ANOVA).

Extracellular concentrations of brain lactate in ALF demonstrated a similar pattern as that observed with extracellular brain ammonia, where extracellular brain lactate was significantly higher than shams at $\boldsymbol{T}=2$ ( 2 hours following liver devascularization; Fig. 2A). As early as 1 hour following MARS treatment, extracellular brain lactate normalized, which persisted until $\boldsymbol{T}=6$ hours. 

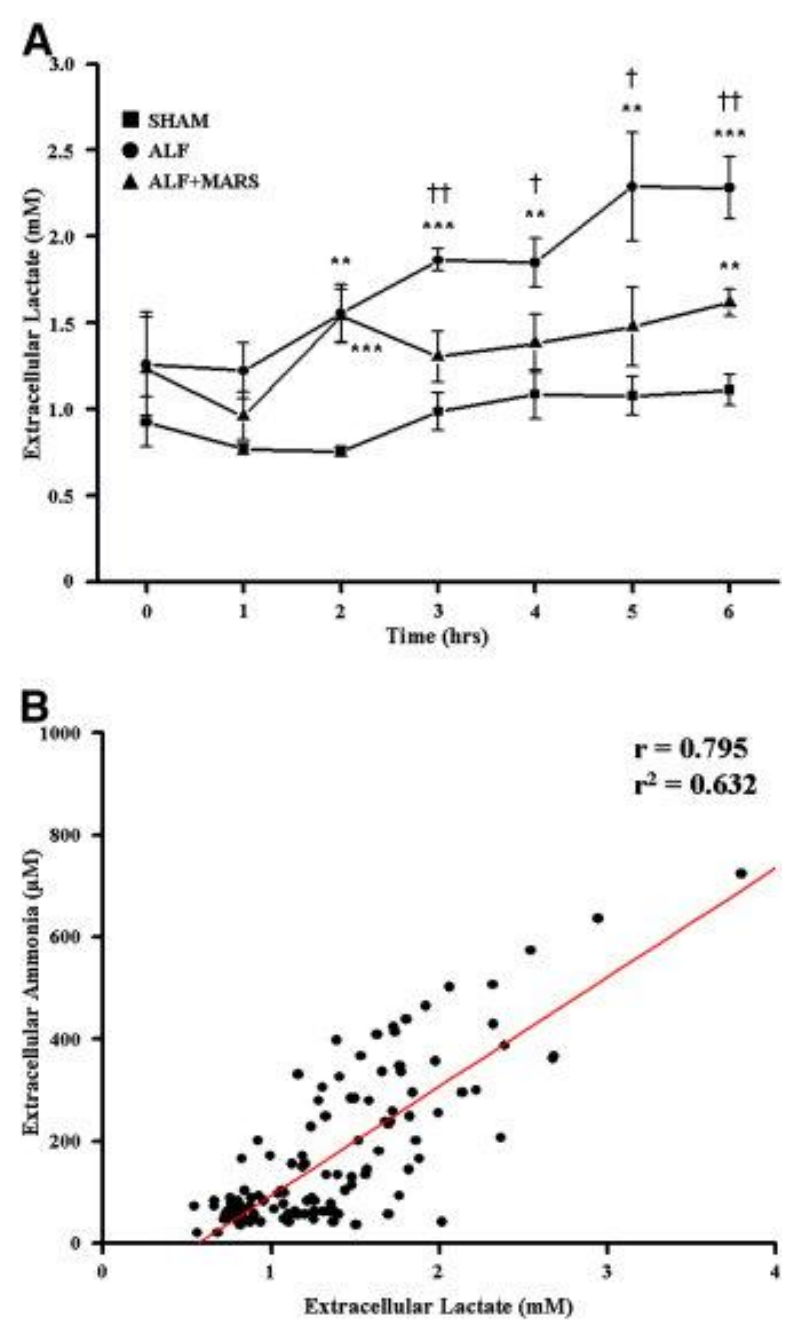

Figure 2. (A) Extracellular concentrations of lactate following induction of $\operatorname{ALF}(\boldsymbol{T}=0)$. (B) Correlation between extracellular brain ammonia and lactate $\left(* * * \boldsymbol{P}<0.001,{ }^{* *} \boldsymbol{P}<0.01\right.$ versus SHAM; ${ }^{\dagger} \boldsymbol{P}<0.01,{ }^{\dagger} \boldsymbol{P}$ $<0.05$, ALF versus MARS; 2-way ANOVA).

Using all 6 times from all 3 groups, a significant correlation was found between extracellular brain ammonia and extracellular brain lactate concentrations $\left(\boldsymbol{r}^{2}=0.632, \boldsymbol{P}<0.001\right.$; Fig. 2B). No significant correlations were found between percent change in ICP and percent change in extracellular brain lactate (data not shown).

\section{Arterial Lactate.}

Arterial lactate concentrations were significantly increased in ALF and ALF+MARS at all times-2, 4, and 6 hourscompared to in the sham pigs (Fig. 3). No significant differences were found between ALF and ALF+MARS at any time. 


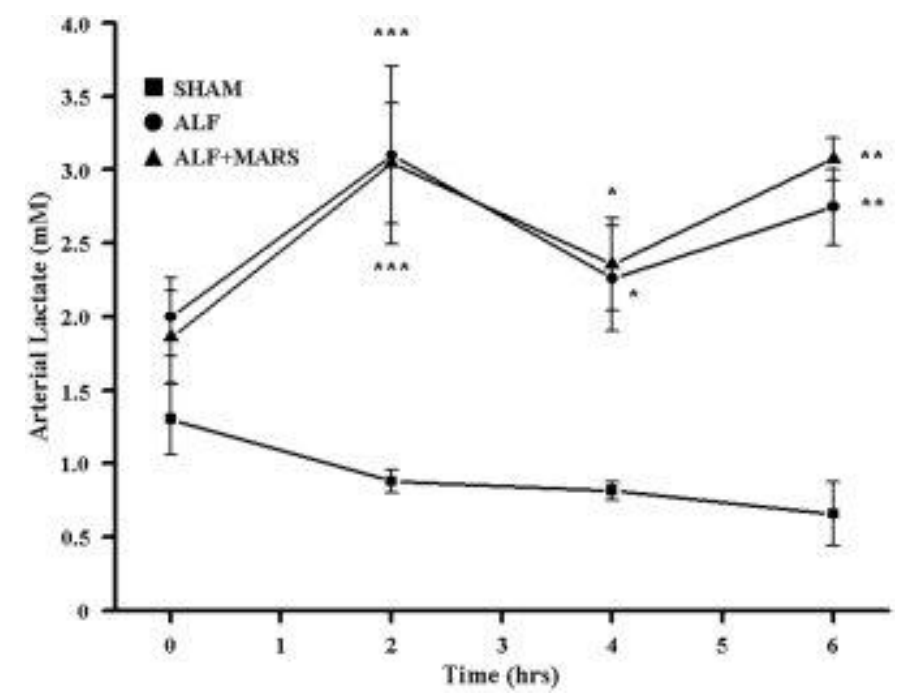

Figure 3. Arterial concentrations of lactate following induction of ALF at $\boldsymbol{T}=0(* * * \boldsymbol{P}<0.001, * * \boldsymbol{P}<0.01$, $* \boldsymbol{P}<0.05$ versus SHAM; 1-way ANOVA).

\section{Arterial and Extracellular Brain Glucose.}

Arterial glucose levels were closely monitored and controlled to remain within physiological concentrations in all 3 groups (Table 1). No significant changes were found in extracellular brain glucose concentration in any of the 3 groups over the 6-hour duration of the experiment (Table 1).

Table 1. Concentrations of Arterial Glucose, Extracellular Brain Glucose, and Extracellular Brain Pyruvate Following Induction of ALF

\begin{tabular}{|c|c|c|c|c|c|c|c|}
\hline & \multicolumn{7}{|c|}{ Time (hours) } \\
\hline \multicolumn{8}{|c|}{ Arterial glucose (mM) } \\
\hline ALF & $4.30 \pm 0.88$ & $5.22 \pm 0.64$ & $5.32 \pm 0.49$ & $4.05 \pm 0.22$ & $5.25 \pm 0.84$ & $3.69 \pm 0.32$ & $4.03 \pm 0.49$ \\
\hline ALF+MARS & $4.23 \pm 0.81$ & $5.96 \pm 1.05$ & $5.58 \pm 0.51$ & $4.53 \pm 0.48$ & $4.83 \pm 0.20$ & $4.79 \pm 0.19$ & $4.73 \pm 0.25$ \\
\hline ALF & $1.77 \pm 0.17$ & $2.35 \pm 0.64$ & $2.24 \pm 0.21$ & $2.32 \pm 0.35$ & $2.18 \pm 0.36$ & $1.76 \pm 0.33$ & $1.56 \pm 0.28$ \\
\hline$A L F+M A R S$ & $2.04 \pm 0.39$ & $3.11 \pm 0.91$ & $2.70 \pm 0.46$ & $2.42 \pm 0.51$ & $2.49 \pm 0.54$ & $2.20 \pm 0.35$ & $1.90 \pm 0.32$ \\
\hline \multicolumn{8}{|c|}{ Extracellular brain pyruvate $(\mu \mathrm{M})$} \\
\hline SHAM & $78.14 \pm 4.68$ & $69.39 \pm 6.10$ & $72.37 \pm 7.20$ & $91.28 \pm 14.51$ & $101.15 \pm 14.24$ & $101.86 \pm 13.28$ & $101.73 \pm 10.20$ \\
\hline
\end{tabular}

\section{Extracellular Brain Pyruvate.}

No significant changes were measured in extracellular brain pyruvate in any of the 3 groups over the 6-hour duration of the experiment (Table 1).

\section{Ratios of Brain Energy Metabolites.}

Ratio of extracellular brain lactate/pyruvate, a measure of anaerobic metabolism, 27 was found to be significantly increased at $\boldsymbol{T}=6$ hours in the ALF group compared with the sham group $(\boldsymbol{P}<0.05 ;$ Fig. 4A). At the same time, a 4-hour MARS treatment attenuated this increase. 

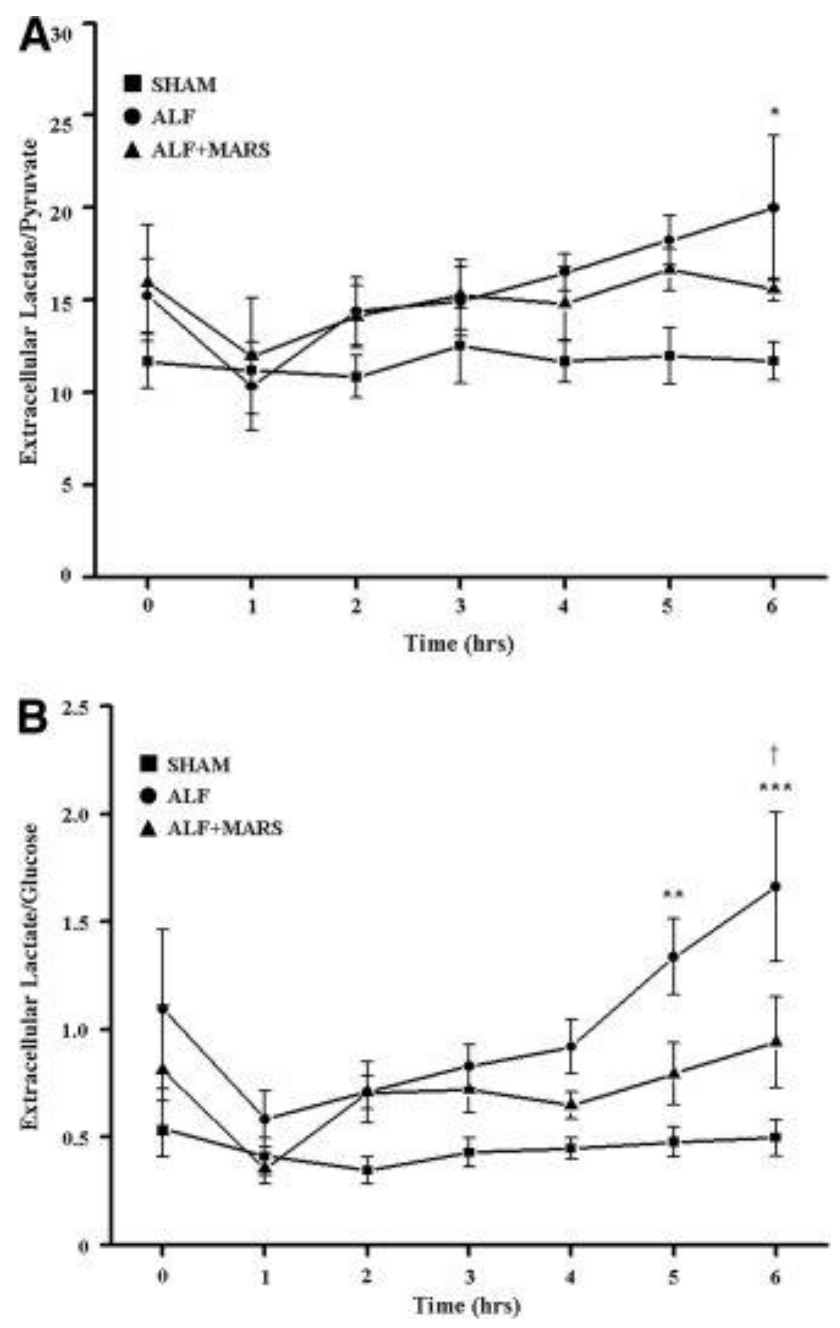

Figure 4. (A) Extracellular brain lactate/pyruvate ratio and (B) extracellular brain lactate/glucose ratio following induction of ALF at $\boldsymbol{T}=0\left(* * * \boldsymbol{P}<0.001, * * \boldsymbol{P}<0.01, * \boldsymbol{P}<0.05\right.$, versus SHAM; ${ }^{\dagger} \boldsymbol{P}<0.05$, ALF versus MARS; 2-way ANOVA).

A significant increase in the ratio of extracellular brain lactate/glucose (indicating accelerated glycolysis) was found in the ALF group versus the sham group at $\boldsymbol{T}=5$ hours $(\boldsymbol{P}<0.01)$ and $\boldsymbol{T}=6$ hours $(\boldsymbol{P}<0.001)$. A 4-hour treatment of MARS resulted in normalization at $\boldsymbol{T}=6$ hours $(\boldsymbol{P}<0.05$; Fig. 4B).

\section{Extracellular Brain Glutamate, Glutamine.}

Extracellular brain glutamate was significantly increased in the ALF group at $\boldsymbol{T}=5$ hours $(\boldsymbol{P}<0.01)$ and $\boldsymbol{T}=6$ hours $(\boldsymbol{P}$ $<0.01)$ compared to the sham group and normalized at the same times with MARS following 3 hours $(\boldsymbol{P}<0.05)$ and 4 hours $(\boldsymbol{P}<0.001)$ of treatment (Fig. 5A). Extracellular brain glutamine was significantly increased in the ALF group compared to the sham group at $\boldsymbol{T}=3-6$ hours $(\boldsymbol{P}<0.05)$. MARS treatment had no effect on extracellular brain glutamine, as a significant increase was also found in the ALF+MARS group compared to the sham group, however, only from $\boldsymbol{T}=$ 4 to 6 hours $(\boldsymbol{P}<0.01$; Fig. 6B). No significant correlations were found between percent change in ICP and percent change in either extracellular brain glutamate or glutamine (data not shown). 

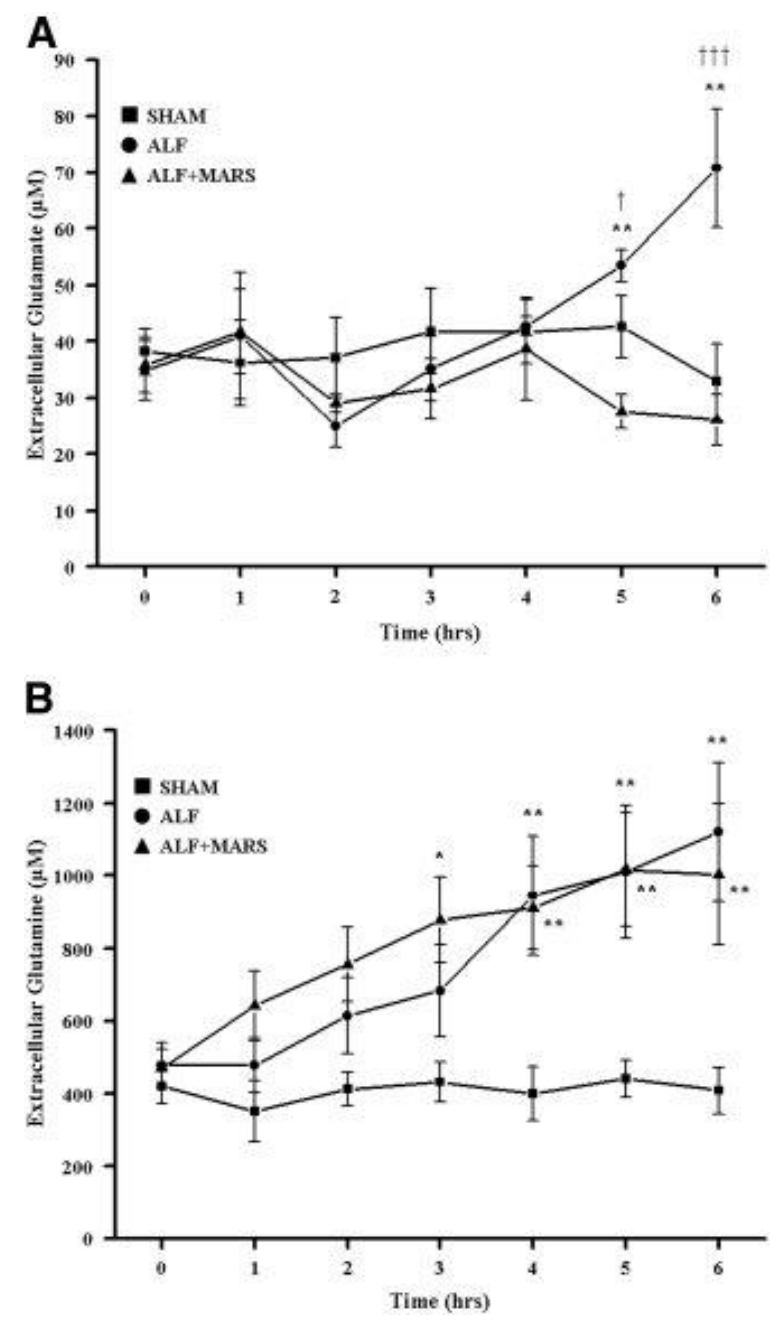

Figure 5. Extracellular brain concentrations of (A) glutamate and (B) glutamine following induction of ALF at $\boldsymbol{T}=$ $0\left({ }^{* *} \boldsymbol{P}<0.01,{ }^{*} \boldsymbol{P}<0.05\right.$ versus SHAM; ${ }^{\dagger \dagger} \boldsymbol{P}<0.01,{ }^{\dagger} \boldsymbol{P}<0.05$, ALF versus MARS; 2 -way ANOVA).

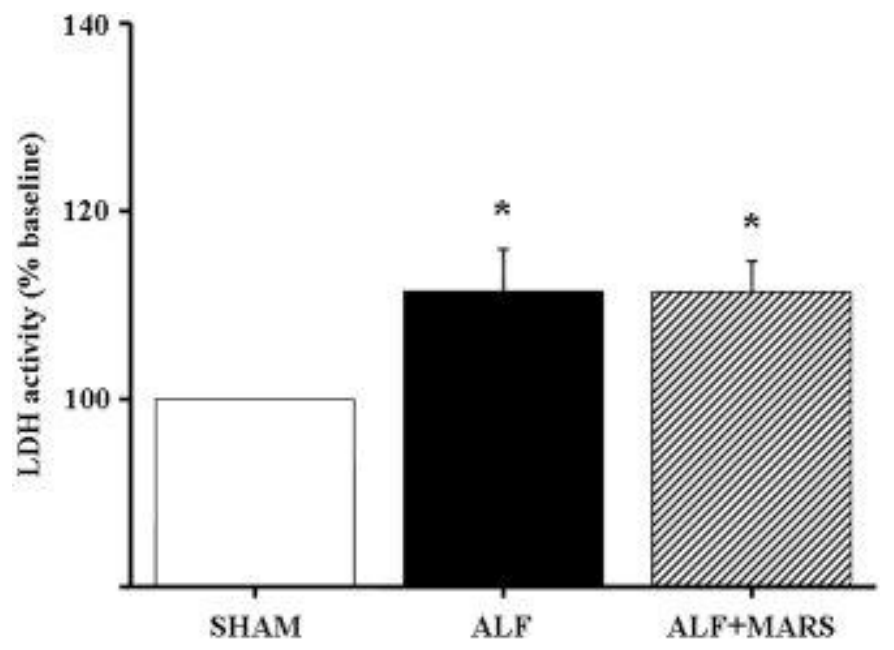

Figure 6. LDH activity (\% baseline) in frontal cortex following induction of ALF at $\boldsymbol{T}=0\left({ }^{*} \boldsymbol{P}<0.05\right.$ versus SHAM; 1-way ANOVA). 


\section{Lactate Dehydrogenase Activity in Brain.}

An increase in LDH activity was found in the frontal cortex in pigs with ALF compared to sham pigs. This same increase was found following a 4-hour MARS treatment (Fig. 6).

\section{Oxygen Extraction.}

The calculated oxygen extraction showed no significant change in oxygen uptake across the brain in all 3 groups at $\boldsymbol{T}=6$ hours (sham: $0.53 \pm 0.03$; ALF: $0.55 \pm 0.02$; ALF+MARS: $0.57 \pm 0.03$ ).

\section{Mitochondrial Enzyme Activity.}

No differences were found between the groups in the activity levels of mitochondrial electron transport chain complexes I or II/III (Fig. 7A,B). A significant increase in the activity of complex IV (cytochrome $c$ oxidase) was found in brain tissue (frontal cortex) homogenates from pigs in both the ALF and ALF+MARS groups compared to sham animals $(\boldsymbol{P}<0.05$ for both, Fig. 7C). This finding was reflected in the increased level of cytochrome $c$ oxidase protein measured in the homogenate, which was likewise significantly increased in both test groups compared to the sham-operated control $(\boldsymbol{P}<$ 0.05 for both, Fig. 7D).
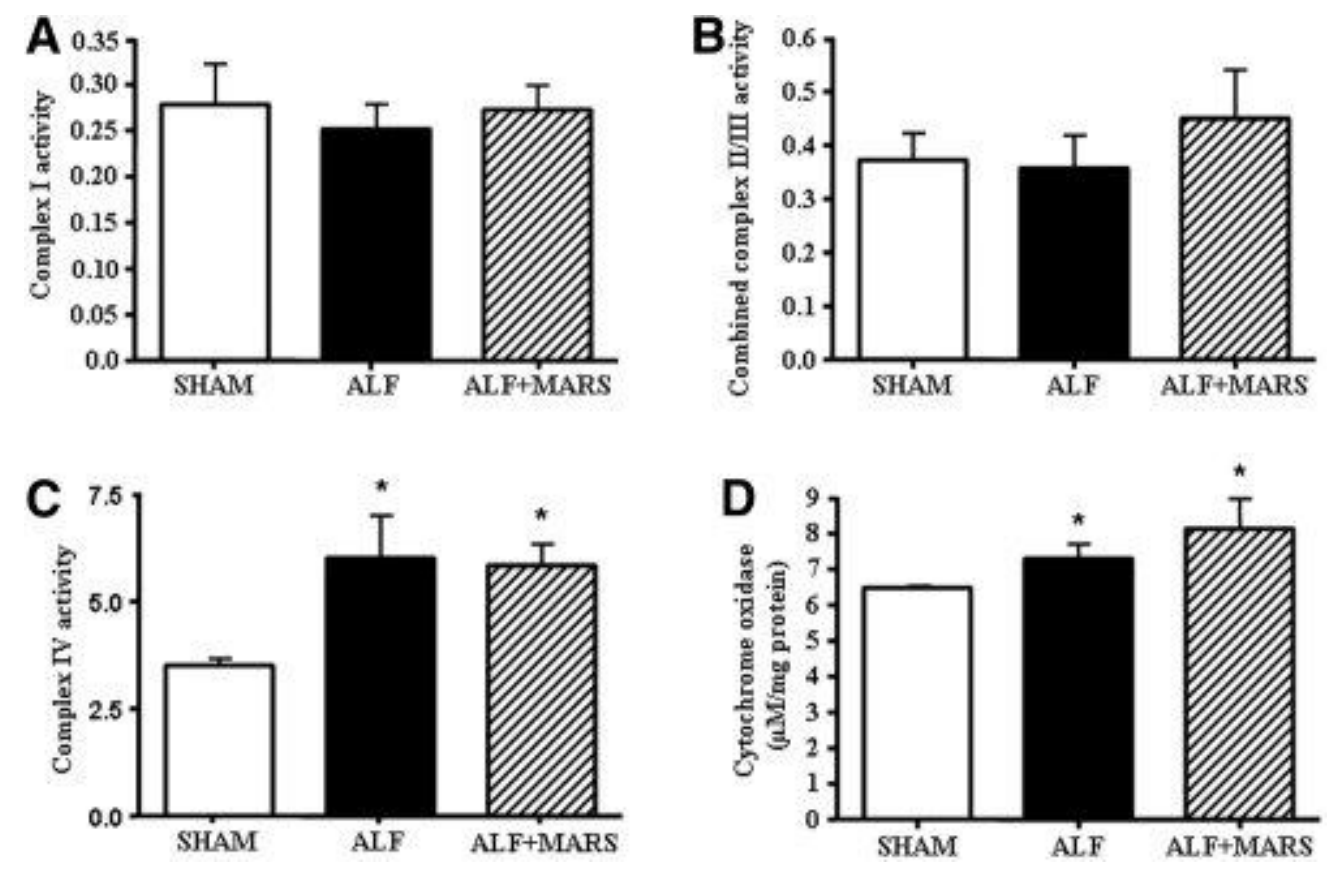

Figure 7. (A) Mitochondrial complex I activity relative to citrate synthase activity, expressed as the rotenone-sensitive rate; (B) complex II/III combined activity relative to citrate synthase activity, expressed as the antimycin A-sensitive rate; (C) complex IV activity assay relative to citrate synthase activity, expressed as the cyanide-sensitive rate; (D) concentration of complex IV in brain tissue homogenate ( $* \boldsymbol{P}<0.05$ versus SHAM; 1-way ANOVA).

\section{DISCUSSION}

We previously demonstrated in pigs with ALF that a 4-hour albumin dialysis (MARS) treatment results in attenuation of increased ICP. ${ }^{17}$ We also observed that this reduction in ICP was independent of changes in the currently proposed pathophysiological mechanisms: arterial ammonia, cerebral blood flow, and inflammation. Hence, this experimental paradigm allows testing for alternative hypotheses. The results of this study reveal that the development of intracranial hypertension in ALF is associated with increased extracellular brain ammonia, lactate, and glutamate, with a significant correlation found between extracellular brain ammonia and lactate. The reduction in these metabolites in the MARS- 
treated animals in association with reduced ICP indicates the important role of these metabolites in the pathogenesis of intracranial hypertension in ALF.

Our study provides novel data that indicate the importance of extracellular ammonia in the pathogenesis of increased ICP in ALF, including a correlation between percent change in extracellular brain ammonia and percent change in ICP.

Previous studies have suggested that the rate of ammonia delivery to the brain correlates with the severity of intracranial hypertension in patients with ALF. ${ }^{7}$ Our study provides further insights into the relationship between arterial and extracellular brain ammonia. As early as 2 hours following ALF, pigs developed a significant increase in extracellular brain ammonia in the brain. From the beginning of the MARS treatment, at $\boldsymbol{T}=2$ hours, until $\boldsymbol{T}=6$ hours, extracellular brain ammonia rose $244 \%$ in the ALF pigs compared to $80 \%$ in the MARS-treated ALF pigs. This attenuation in the extracellular compartment of the brain in the MARS-treated animals (and no change in arterial ammonia ${ }^{17}$ ) depicts the importance of extracellular brain ammonia in the pathogenesis of intracranial hypertension in ALF.

Changes in arterial and extracellular brain lactate occurred in a pattern similar to that of ammonia. ${ }^{17}$ An increase in extracellular brain and arterial lactate was observed in ALF pigs; however, only extracellular brain lactate was normalized following MARS treatment, as increased arterial lactate remained unaffected. This indicates that cerebral, and not arterial, lactate is likely to be more important in the pathophysiology of increased ICP in ALF. Our results are in keeping with similar observations in patients with ALF but argue against the suggestion that the increase in extracellular brain lactate is blood-borne rather than produced in the brain. ${ }^{28}$ Lactate is produced by the action of LDH, an enzyme in neurons and astrocytes that is implicated during anaerobic glycolysis/TCA cycle inhibition. Our data showing an increase in LDH activity in the pigs with ALF compared to sham-operated animals suggests that lactate production is occurring in the brain. An increase in extracellular brain lactate may result from an increase in anaerobic metabolism because of either cerebral hypoxia or inhibition of aerobic energy metabolism. The attenuation of the increased lactate:glucose and lactate:pyruvate ratios (with no change in extracellular brain glucose or pyruvate) in the ALF animals treated with MARS is the result of a reduction in extracellular brain lactate in the same animals. Interestingly, LDH activity was not attenuated in the MARS-treated pigs. The mechanism for this remains unclear; however, a decrease in extracellular lactate would result from increased removal or decreased utilization of lactate from the extracellular space. Lactate is not only an energy impairment marker but also an energy source for neurons. ${ }^{29}$ This would entail neurons removing lactate more efficiently in the MARS-treated pigs.

To investigate whether the increase in lactate was a result of inhibition of aerobic energy metabolism, we measured the activity of mitochondrial enzyme complexes of the electron transport chain in the frontal cortex. We observed that complex IV activity was increased together with cytochrome oxidase in both the ALF- and ALF+MARS-treated animals. The activity of the other enzymes remained unchanged in all 3 groups. These data contrast with those of previous studies of cultured astrocytes exposed to $5 \mathrm{mM}$ concentrations of ammonia, in which mitochondrial proteins (in particular, complex IV) were found to be reduced following long-term exposure. ${ }^{30-32}$ The maintenance of normal oxygen consumption further provides evidence that mitochondrial function is maintained in the brain during ALF.

It is known that elevated ammonia concentrations inhibit $\alpha$-ketoglutarate dehydrogenase ${ }^{6}$ and reduce energy availability. ${ }^{33}$ A strong correlation between extracellular brain ammonia and extracellular brain lactate supports the hypothesis that ammonia may stimulate increased lactate. This increase in lactate may indicate a situation of energy deficit due to an imbalance between energy demand and supply. Brain detoxifies ammonia into glutamine by an energy-requiring process involving glutamine synthetase. Furthermore, ammonia and glutamine synthetase activity stimulate an increase in glutamate uptake into astrocytes. ${ }^{9,34}$ This, in addition to glutamate-activated and/or ammonia-induced NMDA receptor activation, stimulates the ATP-required $\mathrm{Na}^{+}-\mathrm{K}^{+}$ATPase. Therefore, ammonia may affect brain energy metabolism and increase extracellular brain lactate by increasing the demand for and impairing the supply of ATP. The correlation between extracellular brain ammonia and extracellular brain lactate along with a reduction of both in association with ICP attenuation in MARS-treated pigs further supports this hypothesis. 
An increase in extracellular brain glutamate has been consistently demonstrated in different animal models of ALF, ${ }^{24,35-38}$ which could be the result of decreased glutamate uptake or increased glutamate release. ${ }^{39}$ It has been recently demonstrated that an acute insult of ammonia leads to glutamate release from astrocytes. ${ }^{40}$ Accordingly, we observed an increase in extracellular brain glutamate in the ALF animals, which was normalized with MARS treatment. Glutamate uptake by high-affinity glutamate transporters ${ }^{41}$ found in astrocytes stimulates $\mathrm{Na}^{+} / \mathrm{K}^{+}$ATPase, which increases aerobic glycolytic lactate production that is then taken up by neurons and used for oxidative phosphorylation. ${ }^{29}$ According to this hypothesis, a disproportionate amount of glycolysis may occur despite sufficient oxygen levels. ${ }^{42}$ The importance of extracellular brain glutamate in the pathogenesis of increased ICP is indicated by the observation that MARS-induced normalization of extracellular brain glutamate was associated with attenuation of the rise in ICP. The lack of correlation between extracellular brain glutamate and ICP in a study of patients with ALF may indicate that those patients were at a later stage of the disease. ${ }^{28}$

Pigs with ALF showed an increase in extracellular brain glutamine that was unaffected by MARS treatment, supporting the view that extracellular brain glutamine is not likely to be important in increased ICP in ALF. These data are in contrast to those from a study of patients with ALF that showed extracellular brain glutamine was related to surges in ICP. ${ }^{43}$ This may be because we are studying the initiating mechanisms of intracranial hypertension in ALF.

In summary, our results demonstrate that percent change in ICP is correlated with percent change in extracellular brain ammonia in pigs with ALF, which is associated with changes in extracellular lactate and glutamate but not glutamine. The attenuation of increased extracellular brain ammonia in the MARS-treated animals may reduce energy demand (by lowering extracellular brain glutamate) and increase supply, leading to attenuation of the increase in lactate. The observation that brain oxygen consumption and mitochondrial complex enzyme activity changed in parallel in both ALFand MARS-treated animals supports this hypothesis. In conclusion, the results of our study indicate an important role for extracellular brain ammonia in the pathogenesis of intracranial hypertension in ALF, but how MARS reduces extracellular brain ammonia requires further investigation.

\section{Acknowledgements}

Teraklin AG provided MARS kits.

\section{REFERENCES}

1 Jalan R. Intracranial hypertension in acute liver failure: pathophysiological basis of rational management. Semin Liver Dis 2003; 23: 271-282.

2 Jalan R, Olde Damink SW, Hayes PC, Deutz NE, Lee A. Pathogenesis of intracranial hypertension in acute liver failure: inflammation, ammonia and cerebral blood flow. J Hepatol 2004; 41: 613-620.

3 Felipo V, Butterworth RF. Neurobiology of ammonia. Prog Neurobiol 2002; 67: 259-279.

4 Clemmesen JO, Larsen FS, Kondrup J, Hansen BA, Ott P. Cerebral herniation in patients with acute liver failure is correlated with arterial ammonia concentration. HEPATOLOGY 1999; 29: 648-653.

5 Dejong CH, Deutz NE, Soeters P. Cerebral cortex ammonia and glutamine metabolism in two rat models of chronic liver deficiency-induced hyperammonemia: influence of pair-feeding. J Neurochem 1993; 60: 1047-1057.

6 Lai JC, Cooper AJ. Brain alpha-ketoglutarate dehydrogenase complex: kinetic properties, regional distribution, and effects of inhibitors. J Neurochem 1986; 47: 1376-1386.

7 Chatauret N, Zwingmann C, Rose C, Leibfritz D, Butterworth RF. Effects of hypothermia on brain glucose metabolism in acute liver failure: a H/C-nuclear magnetic study. Gastroenterology 2003; 125: 815-824.

8 Zwingmann C, Chatauret N, Leibfritz D, Butterworth RF. Selective increase of brain lactate synthesis in experimental acute liver failure: results of a [H-C] nuclear magnetic study. HEPATOLOGY 2003; 37: 420-428.

9 Mort D, Marcaggi P, Grant J, Attwell D. Effect of acute exposure to ammonia on glutamate transport in glial cells isolated from the salamander retina. J Neurophysiology 2001; 86: 836-844. 
10 Kosenko E, Kaminsky Y, Grau E, Miñana MD, Marcaida G, Grisolia S, et al. Brain ATP depletion induced by acute ammonia intoxication in rats is mediated by activation of the NMDA receptor and $\mathrm{Na}^{+}-\mathrm{K}^{+}$-ATPase. J Neurochem 1994; 63: 2172-2178.

11 Tsai MH, Chen YC, Wu CS, Ho YP, Fang JT, Lien JM, et al. Extracorporeal liver support with molecular adsorbents recirculating system in patients with hepatitis B-associated fulminant hepatic failure. Int J Clin Pract 2005; 59: 12891294.

12 Sen S, Ytrebo LM, Rose C, Fuskevaag OM, Davies NA, Nedredal GI, et al. Albumin dialysis: a new therapeutic strategy for intoxication from protein-bound drugs. Intensive Care Med 2004; 30: 496-501.

13 Hassanein T, Tofteng F, Brown AS, McGuire BM, Lynch P, Larsen FS, et al. Efficacy of albumin dialysis (MARS) in patients with cirrhosis and advanced grades of hepatic encephalopathy: A prospective, controlled, randomized multicenter trial. [abstract] HEPATOLOGY 2004; 40(4 suppl. 1 ): 726A-727A.

14 Heeman U, Triechel U, Loock J, Philip T, Gerken G, Malago, Klammt S, Loehr M, Liebe S, Mitzner S, Schmidt R, Stange J. Albumin dialysis in cirrhosis with superimposed acute liver injury: a prospective, controlled study. HEPATOLOGY 2002; 36(4 pt 1): 949-958.

15 Mitzner SR, Stange J, Klammt S, Peszynski P, Schmidt R, Noldge-Schomburg G. Extracorporeal detoxification using the molecular adsorbent recirculating systemfor critically ill patients with liver failure. J Am Soc Nephrol 2001; 12: S75S82.

16 Sorkine P, Ben Abraham R, Szold O, Biderman P, Kidron A, Merchav H, et al. Role of the molecular adsorbent recycling system (MARS) in the treatment of patients with acute exacerbation of chronic liver failure. Crit Care Med 2001; 29: 1332-1336.

17 Sen S, Rose C, Ytrebo LM, Davies NA, Nedredal GI, Fuskaveg OM, et al. Effect of albumin dialysis on intracranial pressure increase in pigs with acute liver failure: a randomized study. Crit Care Med 2006; 34: 158-164.

18 Ytrebo LM, Nedredal GI, Langbakk B, Revhaug A. An experimental large animal model for the assessment of bioartificial liver support systems in fulminant hepatic failure. Scand J Gastroenterol 2002; 37: 1077-1088.

19 Ytrebo LM, Korvald C, Nedredal GI, Elvenes OP, Nielsen Grymyr OJ, Revhaug A. N-acetylcysteine increases cerebral perfusion pressure in pigs with fulminant hepatic failure. Crit Care Med 2001: 29; 1989-1995.

20 Ytrebo LM, Ingebrigtsen T, Nedredal GI, Elvenes OP, Korvald C, Romner B, et al. Protein S-100beta: a biochemical marker for increased intracranial pressure in pigs with acute hepatic failure. Scand J Gastroenterol 2000; 35: 546-551.

21 Ten Have GA, Bost MC, Suyk-Wierts JC, van den Bogaard AE, Deutz NE. Simultaneous measurement of metabolic flux in portally-drained viscera, liver, spleen, kidney and hindquarter in the conscious pig. Lab Anim 1996; 30: 347-358. 22 Stange J, Mitzner S. A carrier-mediated transport of toxins in a hybrid membrane. Safety barrier between a patients blood and a bioartificial liver. Int J Artif Organs 1996; 19: 677-691.

23 Stange J, Ramlow W, Mitzner S, Schmidt R, Klinkmann H. Dialysis against a recycled albumin solution enables the removal of albumin-bound toxins. Artif Organs 1993; 17: 809-813.

24 Rose C, Michalak A, Pannunzio M, Chatauret N, Rambaldi A, Butterworth RF. Mild hypothermia delays the onset of coma and prevents brain edema and extracellular brain glutamate accumulation in rats with acute liver failure.

HEPATOLOGY 2000; 31: 872-877.

25 Clark J, Bates T. Investigation of mitochondrial defects in brain and skeletal muscle. In TurnerAJ, BachelardJ, eds. A practical approach to the investigation of metabolic disease. Oxford, UK: IRL Press at Oxford University Press: 151-174. 26 Heales SJR. The biochemical investigation of mitochondrial respiratory chain disorders. CPD Bull Clin Biochem 1999; 1: 78-81.

27 Noske DP, Peerdeman SM, Comans EF, Dirven CM, Knol DL, Girbes AR, et al. Cerebral microdialysis and positron emission tomography after surgery for aneurismal subarachnoid hemorrhage in grade 1 patients. Surg Neurol 2005; 64: $109-115$.

28 Tofteng F, Larsen FS. Monitoring extracellular concentrations of lactate, glutamate, and gycerol by in vivo microdialysis in the brain during liver transplantation in acute liver failure. Liver Transpl 2002; 8: 302-305.

29 Pellerin L, Magistretti PJ. Neuroenergetics: calling upon astrocytes to satisfy hungry neurons. 2004;10: 53-62.

30 Rao KV, Norenberg MD. Cerebral energy metabolism in hepatic encephalopathy and hyperammonemia. Metab Brain Dis 2001; 16: 67-78. 
31 Qureshi K, Rao KV, Qureshi IA. Differential inhibition by hyperammonemia of the electron transport chain enzymes in synaptosomes and non-synaptic mitochondria in ornithine transcarbamylase-deficient spf-mice: restoration by acetyl-1carnitine. Neurochem Res 1998; 23: 855-861.

32 Rao KV, Mawal YR, Qureshi IA. Progressive decrease of cerebral cytochrome C oxidase activity in sparse-fur mice: role of acetyl-L-carnitine in restoring the ammonia-induced cerebral energy depletion. Neurosci Lett 1997; 224: 83-86. 33Ott P, Clemmesen O, Larsen FS. Cerebral metabolic disturbances in the brain during acute liver failure: from hyperammonemia to energy failure and proteolysis. Neurochem Int 2005; 47: 13-18.

34 Hansson E. Primary cultures from defined brain areas. III. Effects of seeding time on [3H]L-glutamate transport and glutamine synthetase activity. Brain Res 1986; 389: 203-209.

35 Bosman DK, Deutz NEP, Maas MAW, van Eijk HMH, Smit JJ, de Hann JG, et al. Amino acid release from cerebral cortex in experimental acute liver failure, studied by in vivo cerebral cortex microdialysis. J Neurochem 1992; 59: 591599.

36 de Knegt RJ, Schalm SW, van der Rijt CCD, Fekkes D, Dalm E, Hekking-Weyma I. Extracellular brain glutamate during acute liver failure and during acute hyperammonemia stimulating acute liver failure: an experimental study based on in vivo brain dialysis. J Hepatol 1994; 20: 19-26.

37 Michalak A, Rose C, Butterworth J, Butterworth RF. Neuroactive amino acides and glutamate (NMDA) receptors in frontal cortex of rats with experimental acute liver failure. HEPATOLOGY 1996; 24: 908-914.

38 Hilgier W, Zielinska M, Borkowska HD, Gadamski R, Walski M, Oja SS, et al. Changes in the extracellular profiles of neuroactive amino acids in the rat striatum at the symptomatic stage of hepatic failure. J Neurosci Res 1999; 56: 7684.

39 Rose C. Effect of ammonia on astrocytic glutamate uptake/release mechanisms. J Neurochem 2006; 97: S11-S15. 40 Rose C, Kresse W, Kettenmann H. Acute insult of ammonia leads to calcium-dependent glutamate release from cultured astrocytes, an effect of pH. J Biol Chem 2005; 280: 20937-20944.

41Lehre KP, Levy LM, Ottersen OP, Storm-Mathisen J, Danbolt NC. Differential expression of two glial glutamate transporters in the rat brain: quantitative and immunocytochemical observations. J Neurosci 1995; 15: 1835-1853. 42 Fox PT, Raichle ME. Focal physiological uncoupling of cerebral blood flow and oxidative metabolism during somatosensory stimulation in human subjects. Proc Natl Acad Sci U S A 1986; 83: 1140-1144.

43Tofteng F, Hauerberg J, Hansen BA, Pedersen CB, Jorgensen L, Larsen FS. Persistent arterial hyperammonemia increases the concentration of glutamine and alanine in the brain and correlates with intracranial pressure in patients with fulminant hepatic failure. J Cereb Blood Flow Metab 2006; 26: 21-27. 